\title{
The effect of stopping before turning on the direct observational measure of whole
}

\section{body turning bias}

MJD Taylor ${ }^{a^{*}} \&$ SC Strike ${ }^{\mathrm{b}}$

${ }^{a}$ University of Essex, School of Biological Sciences, Colchester, Essex, CO4 3SQ, UK

${ }^{\mathrm{b}}$ University of Roehampton, Department of Life Sciences, Whitelands College, Holybourne Avenue, London SW15 4JD, UK

*corresponding author

Email: $\underline{\text { mtaylor@essex.ac.uk }}$

Tel: $+44(0) 1206872818$

\begin{abstract}
Turning bias, the preferential tendency to turn toward a given direction has been reported in both rodents and human participants. The observational gait method of determining turning bias in humans requires a stop prior to turning. This study removed the stop and hypothesised that turning bias would remain the same between stop and non-stop conditions if bias was solely under the control of neurochemical asymmetries. The results showed that statistically turning bias remained the same (to the left) regardless of method used but there was no agreement between the methods thus rejecting the hypothesis. It is likely that when not stopping biomechanical factors related to gait when turning influence the direction of turn rather than solely neurochemical asymmetries.
\end{abstract}

\section{Keywords}

Turning, lateral dominance, biomechanics, gait, bias 


\subsection{Introduction}

Turning bias, the preferential tendency to turn toward a given direction (Mead \& Hampson, 1996) has been reported in both rodents (Zimmerberg, Glick, \& Jerussi, 1974) and human participants (Bracha, Shults, Glick, \& Kleinman, 1987). It is associated with asymmetrical levels of dopamine in the basal ganglia, and preferential turning has been suggested to be toward the hemi-sphere with the least amount of dopamine (Bracha, Shults, et al., 1987). The observational gait method (Yazgan, Leckman, \& Wexler, 1996) is a technique that has been designed to test for turning bias in a laboratory setting. All studies that have employed this method have reported a left population turning bias in normal right handed participants (Glover et al., 2003; Taylor, Strike, \& Dabnichki, 2007; Yazgan et al., 1996; Yazgan, Peterson, Wexler, \& Leckman, 1995). A stipulation of this method is that prior to turning, participant must stop and then turn. There was, however, no justification given why a stop must be implemented. The stipulation of stopping suggests that turning bias maybe altered if stopping is removed, or one could argue whether the constraint of stopping prior to turning is necessary. It was therefore the aim of this study to test Yazgan et al's (1996) method with and without a stop. If turning bias was solely under neurochemical control then one could hypothesise that removing the constraint of stopping will have little effect on turning bias.

\subsection{Methods}

\subsection{Participants}

One hundred healthy participants (47 male, 53 female) mean age ( \pm S.D) $23.8 \pm 8.2$ years volunteered for participation in the study. All participants were free of any head trauma causing dizzy spells/loss of consciousness and free from any neurological illness. Ninetytwo-percent $\left(X^{2}(1, \mathrm{n}=92)=70.56, p=0.0001\right)$ of the participants were right handed as 
assessed by the Lateral Preference Inventory (Coren, 1993). The University granted ethical approval for the study and all participants gave informed consent.

\subsection{Turning bias}

Participants were informed that their gait was being analysed but were not aware of the exact nature of the study. This was because the validity of this type of study relies upon the naivety of the participants where the turn is concerned. Two methods were employed to assess turning bias. The first method (TB1) was adapted from Yazgan et al. (1996). Each subject walked up and down a walkway toward a zone (1.5m deep) $12 \mathrm{~m}$ away, stopping with feet shoulder width apart, turning around, walking back toward the zone at the other end and repeated the procedure ten times. The zones were purely to indicate the end of the walkway and it was hoped that by using a turning zone rather than a mark, targeting would be minimised. The second method (TB2) was similar to the first, except the constraint of stopping before turning was removed. Again, ten trials were collected per participant. The order of TB1 and TB2 were randomised between participants. For each method the direction of turn was recorded, both visually and with a digital video (JVC) recorder.

The spatial environment was controlled in such a way as to prevent external factors influencing turning bias.

i) Participants were only told that their gait was being analysed and only if they asked which way to turn it was strongly suggested that this was not important and they need to do it in their most comfortable way. 
ii) The tester and the video camera were deliberately placed at opposite ends of the line of progression in order to counter balance each other's presence.

iii) The testing space was fully symmetrical - one side of the room was identical to the other.

iv) No lateral illumination in form of either windows or artificial lights was present to avoid influencing the selection process of the turning direction.

v) There were no other ongoing activities in the space.

\subsection{Turning bias scoring}

The percentage of left turns (number of left turns / total number of turns, multiplied by 100) was used to score turning bias. Following the method of Yazgan et al., (1996) a level of 60\% was set as the cut-off for left bias. Scores between $60 \%$ and $100 \%$ indicated a left bias, between $0 \%$ and $40 \%$ indicated a right bias and $50 \%$ no bias. To see if the cut-off level had an effect we also looked at cut-offs at 70\%, 80\%, 90\% and 100\%. For example for the 70\% cut-off; a 100-70\% equals a left bias, $40-60 \%$ is an equal bias, and 30-0\% equals a right bias.

\subsection{Data analysis}

A t-test was used to establish if there was a significant difference between TB1 and TB2, and between males and females. Limits of agreement (Bland \& Altman, 1986) were used to establish the agreement between TB1 and TB2. A chi-squared test was used for exploring the effect of gender on turning bias and used to determine whether the distribution of TB was equal for both TB1 and TB2 at each cut-off. Levels of significance were set at $\mathrm{p}<0.05$ all tests were two-tailed. To test if a significant bias in turning was present in both methods, a one-sample t-test against a value of $50 \%$ was performed on the turning bias scores for each group. 


\subsection{Results}

\subsection{Turning bias}

The average turning bias score for TB1 was $67.5 \pm 38.6 \%$, and for TB2 $62.8 \pm 38.0 \%$, indicating a left population bias for both methods. Statistically there was no significant difference $(t(99)=1.656, p=.105)$ between TB1 and TB2. TB1 and TB2 scores were statistically significantly higher by 17.5 (95\% CI, 9.8 to 25.1 ) and 12.6 (95\% CI, 5.2 to 20.3) respectively than a score of $50 \%(\mathrm{~TB} 1, t(99)=-4.528, p=.0001 ; \mathrm{TB} 2, t(99)=-3.376, p=$ .001 ) for both methods. The limits of agreement showed that TB2 may be $61 \%$ above or $52 \%$ below TB1 score. Forty-three percent of the population altered their turning bias score from TB1 to TB2, of these participants, 22 altered bias . As a population, left bias was more prominent (table 1) during TB1 compared toTB2, and the number of participants reporting an equal bias (table 1) increased when employing the TB2 method. The chi-square goodness-offit test was conducted to determine whether an equal number of the 100 participants chose a specific turning bias direction for each of the cut-off's and for both TB1 and TB2. For each cut-off the minimum expected frequency was 33 . The chi-square test indicated that the number of participants for each cut-off and for both TB1 and TB2 was statistically significantly different with the majority of participants choosing to turn left. As the cut-off for bias increased the change in bias increased from $22 \%$ to $33 \%$. 
The average turning bias score for males (Fig 1) was almost unchanged regardless of turning bias method (TB1 $68.3 \pm 37.1 \%$ vs. TB2 $67.7 \pm 36.1 \%, 95 \%$ CI -6.3 to $7.5,(t(47)=.182, p=$ .857). For females, the average turning bias score was altered more notably from a left population bias to a more equal bias (TB1 $66.7 \pm 40.3 \%$ vs. TB2 $58.2 \pm 39.4 \%$ - Fig 2). This difference $(8.5,95 \% \mathrm{CI}-.55$ to 17.5$)$ however was not statistically significantly $(t(51)=1.884$, $p=.065)$. Of the participants that showed a turning bias for TB1 $(\mathrm{n}=92), 46$ were male and 46 were female. Females were significantly $\left(X^{2} 17.04, p=.0001\right)$ more likely to turn consistently to either the left or right compared to the males $\left(X^{2} 0.783, p=.376\right)$. For TB2 43 females and 42 males reported a turning bias. For the female population this resulted in a reduction of $21 \%$ of the total number of consistent turners compared to TB1. For the male population the total number of consistent turners increased by 1 from TB1 $(n=26)$ to TB2 $(n=27)$.

\subsection{Qualitative description of the turn}

When performing TB1 method, participants employed a generally stereotypical pattern of turning (Fig. 1A). However, even though all participants did walk in a straight line toward the turning zones (from the video recording there was no targeting from the participants), when the constraint of stopping was removed (TB2), three distinct turning strategies were employed (Fig. 1BCD). Firstly a strategy similar to the stop and turn (Fig. 1B) was used minus the actual stop. This was employed by $44 \%$ of the population. Secondly, $41 \%$ of participants turned along an arc (Fig. 1C) in affect lapping in a pattern similar to an 
athletics track. Lastly, $15 \%$ of participants used a pattern similar to the lap method, but on returning to each zone would veer to the opposite corner, thus producing an elongated 'figure of eight' pattern (Fig.1D).

$* * *$ insert fig 1 here***

\subsection{Discussion}

The observational gait method (Yazgan et al., 1996) of turning bias requires a stop before turning. It was hypothesised that if turning bias was solely under neurochemical control then removing the stop would have no effect upon bias. A left population bias was reported for both methods with only a difference of 4.7 in average turning bias scores. Using a cut-off of $60 \%$ a left turning bias was evident in this sample of 100 young adults. This bias remained even when a stricter cut-offs were enforced. Perhaps unsurprisingly as the cut-off increased the number of participants who were classified as equal bias also increased. The left bias reported here is in agreement with other studies using the same method; indeed all studies that have used this method have reported a left population bias in normal subjects (Glover et al., 2003; Taylor et al., 2007; Yazgan et al., 1996, 1995) and in line with those studies using different methods (e.g. Day \& Day, 1997; Lenoir, Van Overschelde, De Rycke, \& Musch, 2006; Toussaint \& Fagard, 2008). However, other studies have reported a right bias or no bias (Bracha, Seitz, Otemaa, \& Glick, 1987; Bracha, 1987; Gospe, Mora, \& Glick, 1990; Mead \& Hampson, 1996, 1997). When dividing the population into sexes, TB1 showed that females were significantly more likely to consistently turn toward a given direction compared to males. This was in agreement with past research (Bracha, Shults, et al., 1987; Mead \& 
Hampson, 1996) and suggests that the female brain is more latearlised than males (Bracha, Shults, et al., 1987).

Even though a significant left bias was seen for both methods, and there was no statistical difference between the two overall bias scores, there was poor agreement between both methods. As such the methods should not be used interchangeable and they may also be under the control of different mechanisms. For example it has been suggested that turning bias is a consequence of neurochemical asymmetries. Yet when removing the 'stop' turning bias altered in $22 \%$ of the population. With bias changing for almost a quarter of the population and no agreement between the two methods this suggests the turning bias is not solely under neurochemical control and that biomechanical factors may play a role. For example when employing TB2, three main turning strategies were identified (Fig. 1BCD). Equal bias almost doubled during TB2, and this was possibly a consequence of the 'figure of 8 ' turning strategy (Fig. 1D), where for example a right turn was used at one end and a left turn at the other. A similar hypothesis for a biomechanical mechanism driving turning bias was put forward by Taylor et al. (2007). They showed that a sample of unilateral trans- tibial amputees showed no preferred tuning bias when performing the same method as TB1 in this present study. Furthermore, turning bias indices in the amputee sample were not significantly associated with lateral dominance, side of amputation, or footedness prior to amputation. However, there was a trend towards turning towards the prosthetic side which allowed the intact limb to act as the stabilising limb during the initiation of the turn. This suggest that turning bias may be overhauled by externally imposed constraints such as amputation (Taylor et al., 2007), by changing the position of the leading foot at the start of the run and turn approach (Lenoir et al., 2006), or as in this present study by removing the stop-and-turn command . 
To state that biomechanics is the only alternative mechanism may, however, be a generalisation. For example, it has also been suggested that turning bias is influenced by vestibular asymmetries (Mohr \& Bracha, 2004; Previc \& Saucedo, 1992), fluctuations of ovarian hormones (Mead \& Hampson, 1997), and visuospatial functioning (Gordon, Busdiecker, \& Bracha, 1992). The method used in this current study is not the only technique employed for establishing turning bias, and other methods (e.g. rotometer and audio methods) have reported different bias results. This suggests that these methods may also not be solely showing neurochemical asymmetries and that external factors play an important role influencing turning bias. For example, Mead \& Hampson, (1996) employed four tape recorders located in each cardinal plane to test turning bias. The test started with a subject facing a tape recorder, upon hearing a 1-second tone the subject turns around and walks toward the tape recorder that emitted the tone. 'Critical trials', those used for turning bias, were the trials where the tone came from directly behind the subject. Similar to the observational gait method, participants were stationary prior to turning; however the population reported a right turning bias. This method (Mead \& Hampson, 1996) relied exclusively upon hearing and so a question regarding any hearing difficulties was asked of the participants. All participants were right-handed and it has been reported that between 63$69 \%$ of all right -handed humans are right-eared (Polemikos \& Papaeliou, 2000; Reiss \& Reiss, 1998) and the duration of hearing is longer in the right ear than the left ear (Dane \& Bayirli, 1998). This suggests the majority of these right-handed participants were possibly right eared, hearing the tone for longer, and so turning toward that side, thus possibly influencing bias. 
Bracha (1987) developed another technique using a rotometer. This is a device worn at belt level for up to 7-hours and uses a compass with magnetic north as its reference. The rotometer records the number of quadrant turns, thus four sequential quadrant turns equal a $360^{\circ}$ turn. Unlike the preceding methods, the rotometer is used in a 'real world' setting and is not laboratory based, thus is designed to measure rotational movement, which is part of a subject's spontaneous activity. This is more akin to the non-stop method used in the current study. It is probable that the studies (Bracha, Seitz, et al., 1987; Bracha, Shults, et al., 1987; Bracha, 1987; Gospe et al., 1990; Mohr, Brugger, Bracha, Landis, \& ViaudDelmon, 2004) that have used this method (rotometer) differ in their population bias due to external/environmental factors. This may also explain why some studies reported equal bias. For example, if a participants enters a room by turning right they will have to exit room by turning left, therefore resulting in equal bias. These studies suggest that tuning bias is either under the control of different mechanisms or the different methodological approaches are testing different aspects of turning bias. This present study has shown that the observational gait method of determining turning bias is affected when stopping is removed before turning. This may imply that this technique of turning bias is not measuring dopamine asymmetries - if this were the case then there would be agreement between the two methods. The reason for this is that other methods have reported different turning bias results and it is also possible that these studies are not reporting solely dopamine asymmetries, as external factors may be more dominant (i.e. biomechanical). However, future work is required to firstly validate these findings on a larger population and a comparative study between turning bias methods is required to establish if these methodologies report the same bias on the same population. 


\section{References}

Bland, J. M., \& Altman, D. G. (1986). Statistical methods for assessing agreement between two methods of clinical measurement. Lancet, 1(8476), 307-10. Retrieved from http://www.ncbi.nlm.nih.gov/pubmed/2868172

Bracha, H. S. (1987). Asymmetric rotational (circling) behavior, a dopamine-related asymmetry: preliminary findings in unmedicated and never-medicated schizophrenic patients. Biological Psychiatry, 22(8), 9951003. Retrieved from http://www.ncbi.nlm.nih.gov/pubmed/3607140

Bracha, H. S., Seitz, D. J., Otemaa, J., \& Glick, S. D. (1987). Rotational movement (circling) in normal humans: sex difference and relationship to hand, foot and eye preference. Brain Research, 411(2), 231-5. Retrieved from http://www.ncbi.nlm.nih.gov/pubmed/3607430

Bracha, H. S., Shults, C., Glick, S. D., \& Kleinman, J. E. (1987). Spontaneous asymmetric circling behavior in hemi-parkinsonism; a human equivalent of the lesioned-circling rodent behavior. Life Sciences, 40(11), 1127-30. Retrieved from http://www.ncbi.nlm.nih.gov/pubmed/3821375

Coren, S. (1993). The lateral preference inventory for measurement of handedness, footedness, eyedness, and earedness: Norms for young adults. Bulletin of the Psychonomic Society, 31(1), 1-3. doi:10.3758/BF03334122

Dane, S., \& Bayirli, M. (1998). Correlations between hand preference and durations of hearing for right and left ears in young healthy subjects. Perceptual and Motor Skills, 86(2), 667-72.

doi:10.2466/pms.1998.86.2.667

Day, H. D., \& Day, K. C. (1997). Directional preferences in the rotational play behaviors of young children. Developmental Psychobiology, 30(3), 213-23. Retrieved from http://www.ncbi.nlm.nih.gov/pubmed/9104552

Glover, D. A., Powers, M. B., Bergman, L., Smits, J. A. J., Telch, M. J., \& Stuber, M. (2003). Urinary dopamine and turn bias in traumatized women with and without PTSD symptoms. Behavioural Brain Research, 144(1-2), 137-41. Retrieved from http://www.ncbi.nlm.nih.gov/pubmed/12946604

Gordon, H. W., Busdiecker, E. C., \& Bracha, H. S. (1992). The relationship between leftward turning bias and visuospatial ability in humans. The International Journal of Neuroscience, 65(1-4), 29-36. Retrieved from http://www.ncbi.nlm.nih.gov/pubmed/1341689

Gospe, S. M., Mora, B. J., \& Glick, S. D. (1990). Measurement of spontaneous rotational movement (circling) in normal children. Journal of Child Neurology, 5(1), 31-4. Retrieved from http://www.ncbi.nlm.nih.gov/pubmed/2299137

Lenoir, M., Van Overschelde, S., De Rycke, M., \& Musch, E. (2006). Intrinsic and extrinsic factors of turning preferences in humans. Neuroscience Letters, 393(2-3), 179-83. doi:10.1016/j.neulet.2005.09.061

Mead, L. A., \& Hampson, E. (1996). A sex difference in turning bias in humans. Behavioural Brain Research, 78(2), 73-9. Retrieved from http://www.ncbi.nlm.nih.gov/pubmed/8864039

Mead, L. A., \& Hampson, E. (1997). Turning bias in humans is influenced by phase of the menstrual cycle. Hormones and Behavior, 31(1), 65-74. doi:10.1006/hbeh.1997.1363

Mohr, C., \& Bracha, H. S. (2004). Compound measure of hand-foot-eye preference masked opposite turning behavior in healthy right-handers and non-right-handers: technical comment on Mohr et al. (2003). Behavioral Neuroscience, 118(5), 1145-6. doi:10.1037/0735-7044.118.5.1145 
Mohr, C., Brugger, P., Bracha, H. S., Landis, T., \& Viaud-Delmon, I. (2004). Human side preferences in three different whole-body movement tasks. Behavioural Brain Research, 151(1-2), 321-6. doi:10.1016/j.bbr.2003.09.006

Polemikos, N., \& Papaeliou, C. (2000). Sidedness preference as an index of organization of laterality. Perceptual and Motor Skills, 91(3 Pt 2), 1083-90. doi:10.2466/pms.2000.91.3f.1083

Previc, F. H., \& Saucedo, J. C. (1992). The relationship between turning behavior and motoric dominance in humans. Perceptual and Motor Skills, 75(3 Pt 1), 935-44. doi:10.2466/pms.1992.75.3.935

Reiss, M., \& Reiss, G. (1998). Ear preference: association with other functional asymmetries of the ears. Perceptual and Motor Skills, 86(2), 399-402. doi:10.2466/pms.1998.86.2.399

Taylor, M. J. D., Strike, S. C., \& Dabnichki, P. (2007). Turning bias and lateral dominance in a sample of ablebodied and amputee participants. Laterality, 12(1), 50-63. doi:10.1080/13576500600892745

Toussaint, Y., \& Fagard, J. (2008). A counterclockwise bias in running. Neuroscience Letters, 442(1), 59-62. doi:10.1016/j.neulet.2008.06.056

Yazgan, M. Y., Leckman, J. F., \& Wexler, B. E. (1996). A direct observational measure of whole body turning bias. Cortex; a Journal Devoted to the Study of the Nervous System and Behavior, 32(1), 173-6. Retrieved from http://www.ncbi.nlm.nih.gov/pubmed/8697747

Yazgan, M. Y., Peterson, B., Wexler, B. E., \& Leckman, J. F. (1995). Behavioral laterality in individuals with Gilles de la Tourette's syndrome and basal ganglia alterations: a preliminary report. Biological Psychiatry, 38(6), 386-90. doi:10.1016/0006-3223(94)00302-J

Zimmerberg, B., Glick, S. D., \& Jerussi, T. P. (1974). Neurochemical correlate of a spatial preference in rats. Science (New York, N.Y.), 185(4151), 623-5. Retrieved from http://www.ncbi.nlm.nih.gov/pubmed/4858234 
Table 1 Percentage of population with a left, right or equal bias for both methods (TB1 and TB2)

\begin{tabular}{|c|c|c|c|c|c|c|c|c|c|c|}
\hline & \multicolumn{2}{|c|}{$60 \%$ cut-off } & \multicolumn{2}{|c|}{$70 \%$ cut-off } & \multicolumn{2}{|c|}{$80 \%$ cut-off } & \multicolumn{2}{|c|}{$90 \%$ cut-off } & \multicolumn{2}{|c|}{$100 \%$ cut-off } \\
\hline & TB1 & TB2 & TB1 & TB2 & TB1 & TB2 & TB1 & TB2 & TB1 & TB2 \\
\hline Left bias & $65 \%$ & $57 \%$ & $60 \%$ & $52 \%$ & $55 \%$ & $46 \%$ & $53 \%$ & $46 \%$ & $47 \%$ & $38 \%$ \\
\hline Right bias & $27 \%$ & $29 \%$ & $23 \%$ & $25 \%$ & $21 \%$ & $20 \%$ & $18 \%$ & $18 \%$ & $16 \%$ & $18 \%$ \\
\hline Equal bias & $8 \%$ & $14 \%$ & $17 \%$ & $23 \%$ & $24 \%$ & $34 \%$ & $29 \%$ & $36 \%$ & $37 \%$ & $44 \%$ \\
\hline Change in bias* & & & & & & & & & & $\%$ \\
\hline
\end{tabular}

*A change in bias is either a change to or from equal bias, or a change to the opposite bias. Change bias was based on cut-off points. For example, $100-60 \%=$ left bias, $50 \%=$ equal bias, and $40-0 \%=$ right bias for the $60 \%$ cut-off, $100-70 \%=$ left bias, $40-60 \%=$ equal bias, and 30-0\% = right bias for the $70 \%$ cut-off point etc. 


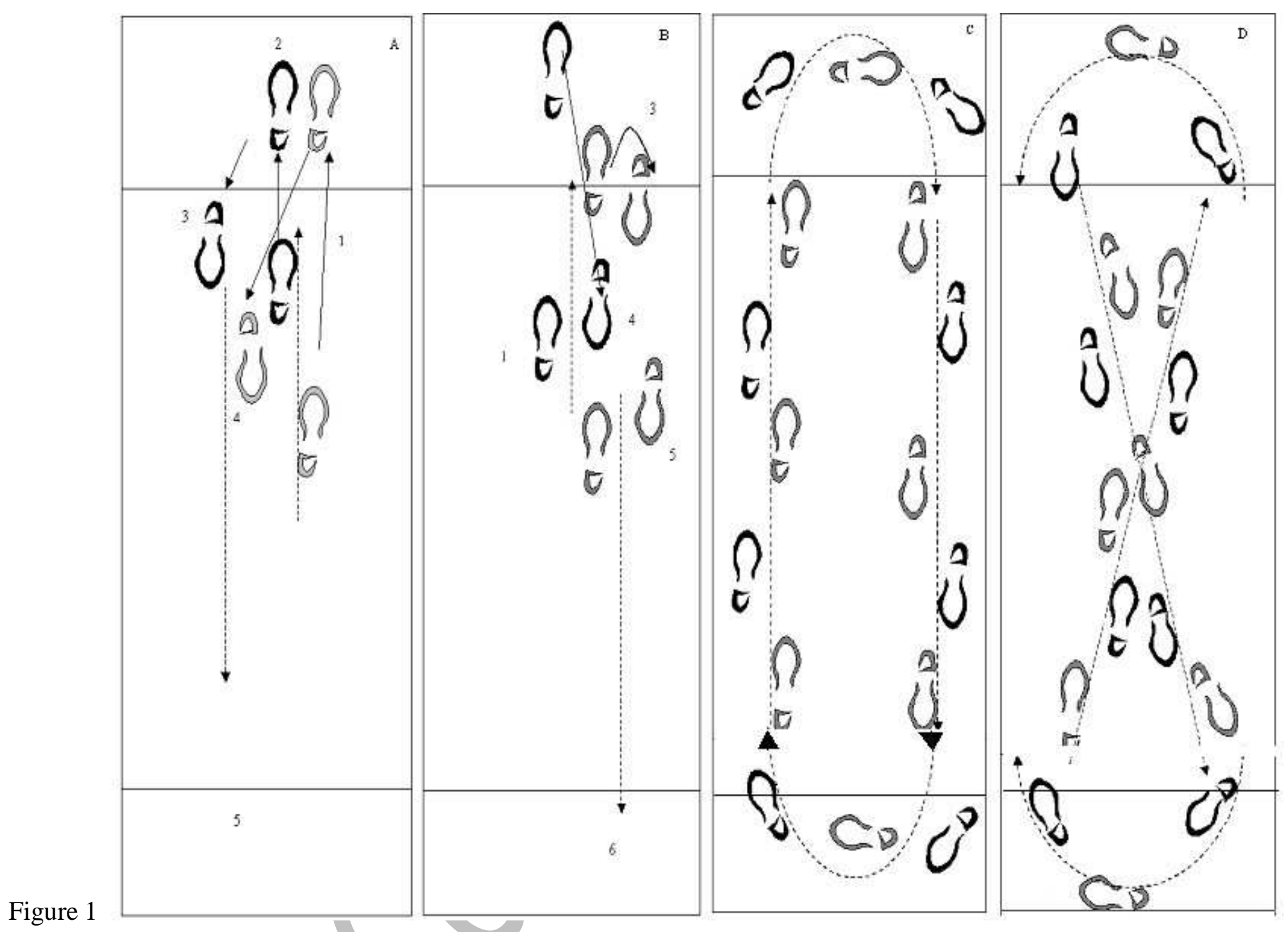


Fig. 1 ABCD. Methods used to turn with the stop - TB1 (A) and when the stop was removed - TB2 $(B, C, D)$. Black feet = left, grey feet $=$ right, solid arrow $=$ swing phase, dashed arrow $=$ direction of progression .

Fig. 1A (TB1): 1) Approach stride, 2) both feet together - stop phase, 3) left foot lifted off and swung into new direction, 4) during left stance (3), the right foot is then swung into the new direction and continues to walk toward the other end and repeat the turn (5). A similar technique was used when turning to the right.

Fig. 1B (TB2): Similar to the stop and turn. 1) Approach stride. 2) Left foot accepts weight in turning zone, and at same time (3) right limb is lifted and rotated, along with the body, toward the right into the new direction. Following rotation, left limb accepts weight and right limb (4) is swung into new direction. 5) Walking toward opposite turning zone where turn is repeated (6).

Fig. 1C (TB2): The 'lap' method, where the turn is completed through an arc in the turning zone. In this example the turn is always to the right at both turning zones.

Fig. 1D (TB2): The 'figure of 8' method. The diagonal approach between zones was subtler than that shown in the figure. An arc was still used in the turning zone but due to the diagonal approach participants turned in opposite directions at either zone (in this example left at the top and right at the bottom), resulting in equal bias.

There were no borders indicating the walkway width as depicted in the figures, the borders here are just for illustrative purposes to separate the four figures. 